Original Article

\title{
Reliability and validity of the Japanese version of the selective control assessment of the lower extremity tool among patients with spastic cerebral palsy
}

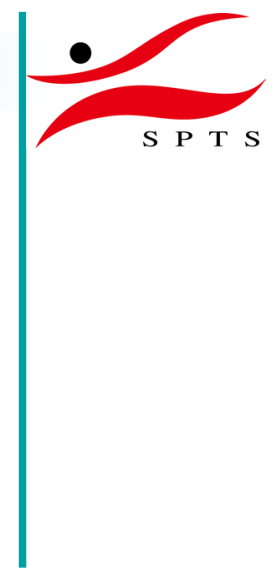

\author{
Yasuaki Kusumoto, PT, $\mathrm{PhD}^{1)^{*}}$, Mami HanaO, MT, MSc ${ }^{2)}$, Kenji TaKaKi, PT, MSc ${ }^{3)}$, \\ Tadamitsu Matsuda, PT, $\mathrm{PhD}^{4}$, Osamu Nitta, PT, PhD ${ }^{5}$ \\ 1) Department of Physicial Therapy, Division of Health Science, Tokyo University of Technology: \\ 5-23-22 Nishikamata, Ohta-ku, Tokyo 144-8635, Japan \\ 2) Department of Medical Technology, Division of Health Science, Tokyo University of Technology, \\ Japan \\ 3) Asukayama Home-visit Nursing Station, Japan \\ 4) Department of Physical Therapy, Faculty of Health Sciences, Uekusa Gakuen University, Japan \\ 5) Department of Physical Therapy, Faculty of Health Sciences, Tokyo Metropolitan University, Japan
}

\begin{abstract}
Purpose] The aim of this study was to translate the Selective Control Assessment of the Lower Extremity (SCALE) tool from English to Japanese and to assess the reliability and validity of the Japanese version of the SCALE (SCALE-J) tool in Japanese patients with spastic cerebral palsy. [Subjects and Methods] The SCALE tool was translated into Japanese in accordance with the published guidelines. In total, 55 patients with spastic cerebral palsy were enrolled in the present study. Reliability by internal consistency (Cronbach's $\alpha$ ), intrarater reliability, inter-rater reliability, and convergent validity by comparing Gross Motor Function Classification System (GMFCS) scores were examined. [Results] The Cronbach's $\alpha$ value of the SCALE-J tool was 0.97-0.98, whereas that of the intrarater and inter-rater reliability ranged from 0.93 to 0.96 . The Spearman correlation coefficient revealed a good relationship between the SCALE tool and the GMFCS. [Conclusion] The SCALE-J tool was found to be reliable and valid; therefore, the SCALE tool may be useful for evaluation in clinical practice.

Key words: Cerebral palsy, Selective voluntary motor control, Reliability
\end{abstract}

(This article was submitted Jun. 29, 2016, and was accepted Aug. 2, 2016)

\section{INTRODUCTION}

Spasticity and contracture in patients with spastic cerebral palsy (CP) may result in apparent impairments, and the lack of selective voluntary motor control (SVMC) of the lower limbs can reportedly lead to severe functional disorders ${ }^{1,2)}$. The evaluation for SVMC of the lower limbs is considered to be an important contributor to the impairment of motor function in patients with $\mathrm{CP}^{2-4)}$. An evaluation of SVMC has been partially performed (i.e., independent dorsiflexion of ankle joint), but a detailed evaluation of SVMC has not yet been performed worldwide.

Fowler developed the Selective Control Assessment of the Lower Extremity (SCALE) tool to clinically examine SVMC in children with spastic $\mathrm{CP}^{5}$ ). The SCALE tool assesses hip, knee, ankle, subtalar, and toe joints bilaterally, and active movements are assigned 0-2 points; the contents of this tool have been assessed for validity, inter-rater reliability, and convergent validity. Moreover, the SCALE tool indicates the relationships of the extensor strength of the knee joint ${ }^{6)}$ and the relationships of the pediatric balance scale ${ }^{7}$. However, in Japan, there are no assessment tools for SVMC of the lower limbs

*Corresponding author. Yasuaki Kusumoto (E-mail: kusumotoys@stf.teu.ac.jp)

(C2016 The Society of Physical Therapy Science. Published by IPEC Inc.

This is an open-access article distributed under the terms of the Creative Commons Attribution Non-Commercial No Derivatives (by-nc-nd) License $<$ http://creativecommons.org/licenses/by-nc-nd/4.0/>. 
among patients with CP. The Gross Motor Function Classification System (GMFCS) is the most used tool for the assessment of motor function classifications for CP. Because the GMFCS varies widely between levels, it is necessary to investigate the characteristics of SVMC per level of the GMFCS.

Therefore, the aim of the present study was to translate the SCALE tool from English into Japanese following the proposed guidelines ${ }^{8)}$ and to assess the reliability and validity of the Japanese version of the SCALE (SCALE-J) tool for Japanese patients with spastic $\mathrm{CP}$.

\section{SUBJECTS AND METHODS}

The SCALE tool is designed to be administered clinically by expert clinicians and consists of three pages: the Directions for Administration, Instructions for Grading, and a Score Sheet ${ }^{5}$. The patient is asked to perform the following movement tasks for a count of 3 seconds: hip flexion and extension, knee extension and flexion, ankle dorsiflexion and plantar flexion with the knee extended, subtalar inversion and eversion, and toe flexion and extension. The SCALE tool assigns each joint a score from 0 to 2 points: 2 points, normal; 1 point, impaired; and 0 points, unable. The SCALE score is the sum of scores for each joint and assumes a 10 point maximum per limb. A rater can perform the evaluation within 15 minutes without specialized equipment after some practice.

Permission to translate the SCALE was granted by the developer (Dr. Eileen G Fowler) before the study was initiated. The translation followed the guidelines proposed by Beaton et $\mathrm{al}^{8)}$. The SCALE was translated from English to Japanese by two independent translators who were native Japanese speakers. One of the translators was blinded to the purpose of the study. The other translator was given information regarding the purpose of the study. The two translations were compared with each other to assess any discordance. The two translators subsequently met to reach a consensus regarding these discordances. This version of the SCALE was then back-translated into English by two native English speakers. The back translations were integrated by a principal investigator when developing the final version of the SCALE-J tool.

Reliability and convergent validity were evaluated using the final version of the SCALE-J tool among the patients with spastic CP, who were recruited from three hospitals in Tokyo and Kanagawa. Fifty-five individuals with spastic CP with GMFCS levels ranging from I to IV were recruited. Eighteen out of these 55 individuals with the same ratio were chosen each for assessment of intrarater reliability and inter-rater reliability. The characteristics of the participants are presented in Table 1. The following inclusion criteria were used: (1) diagnosis of spastic CP, (2) ability to follow simple directions, and (3) age between 6 and 35 years. The following exclusion criteria were used: (1) history of lower extremity musculotendinous transfer or joint fusion and (2) orthopedic intervention or botulinum toxin injection in the lower extremities in the past 6 months.

All patients provided written informed consent. This study was approved by Tokyo University of Technology of Health Sciences Ethical Review Board (Authorization Number: E13H3-023) and Tokyo Metropolitan University of Health Sciences Ethical Review Board (Authorization Number: 14051). It was financially supported by JSPS KAKENHI grant number 26750232. All authors declare that there are no conflicts of interest.

Intrarater reliability of the SCALE-J tool was assessed using the test-retest method by one rater for 18 participants with spastic CP. The rater was a physical therapist with 8 years of experience assessing patients with CP. Internal consistency of the SCALE-J tool was assessed using Cronbach's $\alpha$ value. The intraclass correlation coefficient (ICC 1.1) and corresponding 95\% confidence interval (CI) were calculated for the SCALE scores obtained separately for the left and right limbs.

The inter-rater reliability of the SCALE-J tool was assessed by two raters among 18 participants with spastic CP. The two

Table 1. Participant characteristics and reliability and construct validity

\begin{tabular}{lccc}
\hline & Intrarater reliability & Inter-rater reliability & Construct validity \\
\hline Number & 18 & 18 & 55 \\
Age, yrs (range) & $14.7 \pm 8.2(6-35)$ & $12.6 \pm 5.5(7-28)$ & $14.7 \pm 6.9(6-35)$ \\
Male,n (\%) & $11(61)$ & $12(67)$ & $34(62)$ \\
Distribution of impairments, n (\%) & & & \\
$\quad$ Diplegia & $13(72)$ & $13(72)$ & $41(75)$ \\
Hemiplegia & $1(6)$ & $1(6)$ & $4(7)$ \\
Quadriplegia & $4(22)$ & $4(22)$ & $10(18)$ \\
GMFCS level, n (\%) & & & $15(27)$ \\
I & $5(28)$ & $5(28)$ & $16(29)$ \\
II & $4(22)$ & $4(22)$ & $13(24)$ \\
III & $4(22)$ & $4(22)$ & $11(20)$ \\
IV & $5(28)$ & $5(28)$ &
\end{tabular}

Mean \pm SD. GMFCS: Gross Motor Function Classification System 
raters were physical therapists with 6-8 years of experience assessing patients with CP. Internal consistency of the SCALE was assessed using Cronbach's $\alpha$ value. The intraclass correlation coefficient (ICC 2.1) and the corresponding 95\% CI were calculated for the SCALE scores obtained separately for the left and right limbs.

Fifty-five individuals who had spastic CP with GMFCS levels ranging from I to IV were included for convergent validity. Ten CP patients with a GMFCS level of V were also screened for this study. However, they were excluded because it was difficult to obtain consent for participation and they were not able to complete simple motor movements that they were instructed to perform. Evaluation of convergent validity of the SCALE was performed by one of two therapists who participated in assessment of the inter-rater reliability of the SCALE-J tool. Right and left SCALE scores were summed as a value of the SVMC of the lower extremity. Total SCALE score and GMFCS levels were compared using Spearman's rank correlation coefficients. All analyses were conducted using IBM SPSS Statistics for Windows (version 19.0); $<<0.01$ was considered statistically significant.

\section{RESULTS}

The test of intrarater reliability showed a relatively high ICC. Cronbach's $\alpha$ values and the ICC 1.1 and $95 \%$ CI values for the left and right limbs are presented in Table 2. The Cronbach's $\alpha$ values for SCALE-J ranged from 0.97 to 0.98. ICC 1.1 ranged from 0.94 to 0.96 ( $95 \%$ CI, 0.85-0.99); both ICC 1.1 values were significant $(\mathrm{p}<0.01)$.

The test of inter-rater reliability showed a relatively high ICC. Cronbach's $\alpha$ values and ICC 2.1 and $95 \%$ CI values for the left and right limbs are presented in Table 2. The Cronbach's $\alpha$ values for the SCALE-J were 0.97. ICC 2.1 ranged from 0.93 to 0.94 ( $95 \%$ CI, 0.83-0.98); both ICC 2.1 values were significant $(\mathrm{p}<0.01)$.

SCALE scores and GMFCS levels are presented in Table 3. SCALE scores showed a significant inverse correlation with the GMFCS levels (Spearman's rank correlation coefficient, $-0.87 ; \mathrm{p}<0.01$ ). The mean SCALE score decreased from 14.5 for participants with GMFCS level I to 2.7 in participants with GMFCS level IV. However, the range of the SCALE scores for participants with GMFCS level III was notably larger than the ranges of the SCALE scores of the participants with other levels.

\section{DISCUSSION}

The aim of the present study was to translate the SCALE tool from English to Japanese and to examine the utility of the SCALE-J tool. Translation of this tool did not present any difficulties, and the SCALE-J tool was found to be reliable and valid. The clinical utility of this tool is supported by both high intrarater reliability and high inter-rater reliability. The SCALE tool assessment instructions and rating criteria are clear. However, because the ability to follow simple motor instructions is necessary, patients younger than 6 years and those with severe motor and intellectual disturbances (GMFCS level V) are excluded.

The convergent validity of the SCALE tool showed significant correlation with GMFCS level, which is another measure of disease severity. SVMC is considered important because it is one of the factors leading to functional motility disorder in cases with spastic CP. Sanger et al. defined SVMC as "the ability to isolate the muscle activity in a selected pattern in response to demands of a voluntary motion or posture" ${ }^{\text {") }}$. Damage to the corticospinal tract interferes with the ability to exercise force, control speed, and time muscle contractions and the pattern of voluntary movements; therefore, SVMC may be affected by the presence of spastic CP due to injury to the corticospinal tract, as in the case of injury to periventricular white matter ${ }^{9,10)}$. The convergent validity for the SCALE-J was -0.87 , which is slightly higher than that of the original version of the SCALE

Table 2. Intrarater reliability and inter-rater reliability of SCALE

\begin{tabular}{llccc}
\hline & Limb & Cronbach's $\alpha$ & Reliability & $95 \%$ CI \\
\hline \multirow{2}{*}{ ICC(1.1) } & Right & 0.98 & $0.96^{*}$ & $0.90-0.99$ \\
& Left & 0.97 & $0.94^{*}$ & $0.85-0.98$ \\
ICC(2.1) & Right & 0.97 & $0.94^{*}$ & $0.84-0.98$ \\
& Left & 0.97 & $0.93^{*}$ & $0.83-0.98$ \\
\hline
\end{tabular}

SCALE: Selective Control Assessment of the Lower Extremity. ${ }^{*} \mathrm{p}<0.01$

Table 3. SCALE scores and GMFCS levels

\begin{tabular}{lcccc}
\hline & I & II & III & IV \\
\hline SCALE score & $14.5 \pm 2.3(11-18)$ & $9.6 \pm 2.3(6-13)$ & $5.7 \pm 3.5(1-12)$ & $2.7 \pm 1.5(1-5)$ \\
\hline $\begin{array}{l}\text { Mean } \pm \text { SD (range). SCALE: Selective Control Assessment of the Lower Extremity; GMFCS: Gross Motor Function } \\
\text { Classification System }\end{array}$
\end{tabular}


( -0.83$)$, indicating good validity. Fowler et al. reported that SCALE scores of cases with GMFCS level III ranged from 0 to 13 points, whereas SCALE scores of cases with GMFCS level IV ranged from 0 to 8 points ${ }^{5}$. In contrast, in the present study, the scores for cases with GMFCS level III ranged from 1 to 12 points, whereas the scores for cases with GMFCS level IV ranged from 1 to 5 points. One reason for this discrepancy may be the relatively lower mobility of individuals with GMFCS level III or IV in the present study compared with previous studies. The ranges of SCALE scores for patients with GMFCS level III or IV might vary widely because such patients use walking aids due to spasticity, impaired balance, contractures, deformities, and weakness.

Secondary disabilities such as hip joint or knee joint flexion contractures and equinus deformity are very important issues in $\mathrm{CP}^{11,12}$. Once the relationship between the degree of lower limb deformity and SCALE score is clarified through longitudinal research among spastic $\mathrm{CP}$ cases, it may serve as an important index of preventive physiotherapy intervention. Moreover, the treatment of spasticity can also involve Botox injections and orthopedic surgery. Hence, because spasticity and SVMC are related, the SCALE tool may be used as a pre-assessment before administration of Botox injections or orthopedic surgery for the treatment of spasticity. SVMC can be an important factor contributing to the impairment of motor function in patients with spastic $\mathrm{CP}$.

\section{REFERENCES}

1) Ostensjø S, Carlberg EB, Vøllestad NK: Motor impairments in young children with cerebral palsy: relationship to gross motor function and everyday activities Dev Med Child Neurol, 2004, 46: 580-589. [Medline] [CrossRef]

2) Voorman JM, Dallmeijer AJ, Knol DL, et al.: Prospective longitudinal study of gross motor function in children with cerebral palsy. Arch Phys Med Rehabil, 2007, 88: 871-876. [Medline] [CrossRef]

3) Boyd RN, Graham HK: Objective measurement of clinical findings in the use of botulinum toxin type A for the management of children with cerebral palsy. Eur J Neurol, 1999, 6: 23-35. [CrossRef]

4) Engsberg JR, Ross SA, Collins DR, et al.: Predicting functional change from preintervention measures in selective dorsal rhizotomy. J Neurosurg, 2007, 106 : 282-287. [Medline]

5) Fowler EG, Staudt LA, Greenberg MB, et al.: Selective Control Assessment of the Lower Extremity (SCALE): development, validation, and interrater reliability of a clinical tool for patients with cerebral palsy. Dev Med Child Neurol, 2009, 51: 607-614. [Medline] [CrossRef]

6) Kusumoto Y, Takaki K, Matsuda T, et al.: Relation of selective voluntary motor control of the lower extremity and extensor strength of the knee joint in children with spastic diplegia. J Phys Ther Sci, 2016, 28: 1868-1871. [Medline] [CrossRef]

7) Lim H: Correlation between the selective control assessment of lower extremity and pediatric balance scale scores in children with spastic cerebral palsy. J Phys Ther Sci, 2015, 27: 3645-3649. [Medline] [CrossRef]

8) Beaton DE, Bombardier C, Guillemin F, et al.: Guidelines for the process of cross-cultural adaptation of self-report measures. Spine, 2000, 25: 3186-3191. [Medline] [CrossRef]

9) Sanger TD, Chen D, Delgado MR, et al. Taskforce on childhood motor disorders: definition and classification of negative motor signs in childhood. Pediatrics, 2006, 118: 2159-2167. [Medline] [CrossRef]

10) Staudt M, Pavlova M, Böhm S, et al.: Pyramidal tract damage correlates with motor dysfunction in bilateral periventricular leukomalacia (PVL). Neuropediatrics, 2003, 34: 182-188. [Medline] [CrossRef]

11) Hodgkinson I, Jindrich ML, Duhaut P, et al.: Hip pain in 234 non-ambulatory adolescents and young adults with cerebral palsy: a cross-sectional multicentre study. Dev Med Child Neurol, 2001, 43: 806-808. [Medline] [CrossRef]

12) Park EY, Kim WH: Relationship between activity limitations and participation restriction in school-aged children with cerebral palsy. J Phys Ther Sci, 2015, 27: 2611-2614. [Medline] [CrossRef] 